\title{
Soluciones tecnológicas de las farmacias comunitarias al desabastecimiento de medicamentos: aplicación del modelo colaborativo en red y "big data"
}

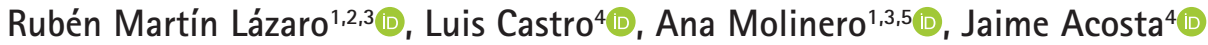 \\ 1. Doctor/a en Farmacia.2. Profesor asociado del departamento de Farmacologia, Farmacognosia y Botánica (Universidad Complutense de Madrid). \\ 3. Farmacéutico/a comunitario/a en Fuenlabrada (Madrid). 4. Farmacéutico comunitario en Madrid. 5. Profesora asociada del departamento de Ciencias \\ Biomédicas (Universidad de Alcalá).
}

\section{PALABRAS CLAVE}

Servicios en farmacia comunitaria, desabastecimiento, tecnología, big data

\section{ABREVIATURAS}

AEMPS: Agencia Española de Medicamentos y Productos

Sanitarios

API: Application Program

Interface

CIMA: Centro de Información de Medicamentos de la AEMPS

CISMED: Centro de Información sobre el Suministro de

Medicamentos

CGCOF: Consejo General de Colegios Oficiales de

Farmacéuticos

GMP: Good Manufacturing

Practice

IIM: Internal Inquirer Manager

LF: Luda Farma ${ }^{\circledR}$

PGEU: Pharmaceutical Group of the European Union (Agrupación Farmacéutica de la Unión Europea)

\section{RESUMEN}

Introducción: la falta de suministro de medicamentos provoca resultados económicos, clínicos y humanísticos negativos en los pacientes, generando también carga de trabajo adicional a los agentes sanitarios.

Para ayudar a los farmacéuticos comunitarios se ha diseñado Luda Farma ${ }^{\circledR}$ (LF), una plataforma electrónica de gestión y colaboración entre farmacias destinada a la mejora de la eficiencia en el control del stock, que proporciona un entorno colaborativo compartiendo información puntual sobre stock de medicamentos concretos entre ellas.

Objetivos: analizar el número de medicamentos en desabastecimiento, según la Agencia Española de Medicamentos y Productos Sanitarios (AEMPS) y el Centro de Información sobre el Suministro de Medicamentos (CISMED) que se localizan para los pacientes a través de la plataforma LF.

Identificar qué medicamentos, que se encuentran en desabastecimiento, se localizan a través de la plataforma.

Material y métodos: estudio retrospectivo, observacional, utilizando los datos generados a través de LF durante el año 2019 (del 1 de enero al 31 de diciembre).

Se utilizó LF para buscar aquellos medicamentos en desabastecimiento según CISMED y AEMPS. Se cruzaron los datos de la red con los listados de CISMED y AEMPS.

El análisis estadístico se realizó con el lenguaje de programación $\mathrm{R}$.

Resultados: participaron 389 farmacias comunitarias adheridas libre y gratuitamente a la red LF.

Realizaron 7.628 encargos de medicamentos: 1.993 con problemas de suministro en AEMPS y 1.794 en CISMED. El resto de los encargos son medicamentos no declarados en falta, en ninguna base de datos y productos que no son medicamentos. Son encargos que realizan las farmacias, ya sea porque no tienen el producto demandado, no pueden ponerlo a disposición del paciente tan rápido como necesita el paciente, aunque lo encarguen, porque se les ha acabado y recurrieron a LF para buscar el producto concreto en otra farmacia para que el paciente pueda recogerlo cuando lo desee el paciente.

Los medicamentos que mayor número de veces se reservaron fueron: Apocard ${ }^{\circledR}$ $100 \mathrm{mg} 60$ comp, Trankimazin Retard ${ }^{\circledR} 0,5 \mathrm{mg} 30$ comp y Elontril ${ }^{\circledR} 150 \mathrm{mg} 30$ comp.

Conclusiones: LF ayuda a los farmacéuticos comunitarios al dar una solución in situ a los pacientes que no encuentran los medicamentos que buscan, disminuyendo el impacto del desabastecimiento de los medicamentos.
Recibido: 15/4/2020

Aceptado: 7/8/2020

Disponible online: $13 / 10 / 2020$
Financiación: ninguna.

Conflicto de intereses: ninguno.

Contribución a la autoría: los autores del estudio hacen constar que el motivo para desarrollar el presente estudio es aclarar el alcance real durante el año de estudio de la herramienta Luda Farma ${ }^{\circledR}$ desde un punto de vista objetivo para conocer su validez sobre los cada vez más acuciantes problemas de suministro de medicamentos en España.

Todos los autores del presente estudio declaran expresamente que el único interés del presente estudio ha sido analizar de manera objetiva los resultados de la implantación y utilización de la red Luda Farma ${ }^{\circledR}$ en las condiciones indicadas en el artículo y no han percibido, ni perciben, ni percibirán, remuneración alguna por el desarrollo y publicación de este.

Cite este artículo como: Martín R, Castro L Molinero A, Acosta J. Soluciones tecnológicas de las farmacias comunitarias al desabastecimiento de medicamentos: aplicación del modelo colaborativo en red y 'big data. Farmacéuticos Comunitarios. 2020 0ct 13; 12 (4): 37-46. doi:10.33620/FC.2173-9218.(2020/Nol12).004.05

Correspondencia: Rubén Martin Lázaro (ruben@farmaciasml.com). 


\section{KEYWORDS}

Community Pharmacy Services, medicine shortage, technology, big data
Technological solutions of community pharmacies to medicines shortage: Application of the collaborative network model and big data

\section{ABSTRACT}

Background: Medicine shortages cause negative economic, clinical and humanistic results for patients, also generating additional workload for healthcare stakeholders.

Luda Farma (LF) is a pharmacy electronic management and collaborative platform aimed at improving efficiency in stock control, and to help pharmacists by providing them a collaborative tool that allows sharing specific information on the stock of particular medications among pharmacies.

Objectives: To analyze the number of medicines in short supply (according to the AEMPS and CISMED listings) that patients locate through the LF platform.

To identify which medicines in short supply are located through the platform.

Method: This retrospective, observational study, used the data generated through LF during the year 2019 (from January 1st to December 31st).

LF was used to search for those medicines in short supply according to CISMED and AEMPS. Data from the network was searched at the CISMED and the AEMPS listings.

Data were processed with programming language $R$.

Results: 389 pharmacies that freely and voluntarily affiliated to the LF network participated.

They carried out 7,628 orders between pharmacies: 1993 of those medicines had supply problems according to the AEMPS, and 1,794 medicines had supply problems according to the CISMED listings.

The drugs with the most significant number of reservations were: Apocard ${ }^{\circledR} 100 \mathrm{mg}$ 60 tablets, Trankimazin Retard ${ }^{\circledR} 0.5 \mathrm{mg} 30$ tablets, and Elontril ${ }^{\circledR} 150 \mathrm{mg} 30$ tablets.

Conclusions: LF helps pharmacists by providing an on-site solution to patients who cannot find the drugs they are looking for, lessening the impact of drug shortages.

\section{Introducción}

Se denomina problema de suministro de un medicamento a la situación en la que las unidades disponibles del mismo en el canal farmacéutico son inferiores a las necesidades de consumo nacional o local (1).

La falta de disponibilidad de medicamentos en la Unión Europea (UE) ha sido un tema de considerable importancia y de preocupación para las autoridades, pacientes y grupos de consumidores, proveedores de atención sanitaria y de la propia industria farmacéutica durante varios años. La indisponibilidad puede surgir debido a tres principales razones:

1. Los medicamentos no están autorizados. Esto se refiere principalmente a medicamentos, cuya disponibilidad es importante para los pacientes, pero para los cuales no se ha presentado la solicitud de comercialización para un estado miembro o el anterior registro válido ha sido retirado.

2. Los medicamentos están autorizados, pero no se comercializan.

3. Las interrupciones de la cadena de suministro impiden directamente la disponibilidad de medicamentos autorizados y comercializados (dificultades de fabricación respecto a normas de correcta fabricación (GMP en inglés), problemas de buena práctica clínica $u$ otros problemas que afectan la calidad de los medicamentos o dan como resultado dudas sobre su seguridad, comercio paralelo o falta de continuidad del suministro dentro de la cadena de medicamento).

Las causas fundamentales de estos motivos son diversas y de alcance multidimensional (2).

La escasez es en algunas ocasiones el resultado de problemas de calidad durante el proceso de fabricación, dando lugar a una parada de la producción para abordar el problema. En el caso de un medicamento con pocos competidores en el mercado, esta interrupción en la producción no puede ser absorbida por otras compañías, y la demanda puede superar la oferta. Además, en el caso de un único fabricante, no existen alternativas para la producción (3).

Las consecuencias en la falta de suministro de los medicamentos las sufren principalmente los pacientes, que pueden experimentar retrasos en el tratamiento, recibir tratamientos alternativos que no sean tan efectivos o bien tolerados (4), o incluso tener que renunciar al tratamiento $(5,6)$. Estos resultados pueden prolongar el sufrimiento del paciente, contribuir a la progresión de la enfermedad y dar lugar a otros resultados de salud adversos que reduzcan el bienestar del paciente y aumenten la morbilidad (7).
Por tanto, puede empeorar los resultados de salud de los pacientes al causar demoras en el tratamiento o cambios en los regímenes de tratamiento, como la sustitución por terapias menos efectivas cuando un medicamento de elección no está disponible. Incluso cuando hay alternativas disponibles al medicamento preferido, la atención del paciente puede verse comprometida (8), pudiendo aparecer problemas de seguridad con la alternativa elegida debidos a la falta de familiaridad con el nuevo medicamento elegido o a la utilización de dosis diferentes.

La falta de medicamentos provoca resultados económicos, clínicos y humanísticos negativos en los pacientes: aumenta la mortalidad, los gastos económicos, el número de errores de medicación, las reacciones adversas $\mathrm{y}$ las quejas de los pacientes, siendo éstas principalmente de frustración y enojo que provocaban un sentimiento de carga del propio paciente hacia sus cuidadores (9). También parece afectar a la adherencia de los pacientes a los tratamientos prescritos (un $60,4 \%$ de los pacientes cuyo medicamento es sustituido por problemas de suministro sufren problemas de adherencia) (10).

Los problemas de desabastecimiento también afectan al resto de los agentes del sistema sanitario, 
suponiendo una sobrecarga para médicos y farmacéuticos comunitarios, que generan costes indirectos debidos a la pérdida de horas en resolver los problemas de suministro, así como a las administraciones sanitarias encargadas de solventarlos con posible sobrecoste, de las alternativas existentes $(11,12)$.

Los pacientes han de desplazarse generalmente para encontrar los medicamentos que necesitan. El 14,7 \% de la población española refiere sufrir una limitación física para andar (13). Así, su capacidad de movilidad limita a "tres manzanas" o 300 metros (14), lo que es poco más de los 250 metros en los que con carácter general pueden encontrar otra farmacia (15). Les resulta difícil ir a distintas farmacias a buscar los medicamentos que precisan, lo cual genera a estos pacientes un inconveniente mayor, comparado con los que no tienen ningún problema para sus desplazamientos.

En España, las situaciones de desabastecimiento pasan por comunicar la falta a la Agencia Española de Medicamentos y Productos Sanitarios (AEMPS), bien por las autoridades sanitarias de las comunidades autónomas, cuando las detectan, o bien por los titulares de la autorización de comercialización del medicamento, que están obligados a informar a la AEMPS sobre cualquier restricción anormal en el suministro de sus medicamentos. La AEMPS publica desde 2008, en su página web, la información de los problemas de suministro de medicamentos de uso humano, manteniendo esta información permanentemente actualizada y ofreciendo una fecha posible de resolución de la misma.

La Organización Farmacéutica Colegial - Colegios Profesionales, Consejos Autonómicos y Consejo General (CGCOF) - ha desarrollado el Centro de Información sobre el Suministro de Medicamentos (CISMED). Las farmacias generan voluntariamente una información que, una vez tratada, permite al colegio de farmacéuticos provincial disponer de datos sobre el suministro de medicamentos en su provincia y, una vez remitida al Consejo General, consolidar los datos a nivel nacional. Así, el CGCOF publica semanalmente listados de medicamentos con problemas de suministro (16).

La Agrupación Farmacéutica de la Unión Europea (PGEU) ha realizado una encuesta anual durante varios años consecutivos para evaluar la progresión de la falta de medicamentos en Europa y comprender su impacto en la práctica farmacéutica comunitaria (17). En 2018, todos los países miembros que respondieron (21) indicaron que habian experimentado desabastecimientos en los 12 meses anteriores, y el $87 \%$ de los países que respondieron indicaron que la situación había empeorado en comparación con el año anterior.

Los farmacéuticos intentan reducir las molestias para los pacientes y el impacto en su atención al mínimo posible. Especialmente, para los medicamentos esenciales, los farmacéuticos comunitarios a menudo aseguran un stock mínimo de una serie de medicamentos muy caros para un paciente individual, asumiendo el riesgo de no poder dispensarlos más cuando ocurren cambios de terapia imprevistos. Los farmacéuticos en Europa gastan en promedio 6,6 horas/semana para mitigar los problemas derivados de la falta de suministro suficiente de medicamentos (12), utilizando medidas, que resultan ineficientes, como grupos de mensajería, llamadas telefónicas, etc., para buscar los medicamentos con falta de disponibilidad.

Para ayudar a los farmacéuticos a paliar los problemas ocasionados a los pacientes por la falta de suministro de los medicamentos se ha diseñado recientemente una solución informática de instalación gratuita basada en inteligencia artificial, Luda Farma ${ }^{\circledR}$ (LF), que ofrece a pacientes y farmacias información en tiempo real sobre las existencias de determinadas referencias disponibles en otras farmacias, y permite conocer al paciente qué farmacia más próxima, o en su zona de elección, tiene el medicamento que necesita (18).

Esta red podría ser de utilidad para mitigar el impacto negativo de los desabastecimientos en nuestra sociedad, y por ello se diseñó este estudio cuyo objetivo principal es analizar el número de medicamentos que se localizan para los pacientes a través de la plataforma LF, y como objetivos secundarios:

- Identificar qué medicamentos, que se encuentran en desabastecimiento, se localizan a través de la plataforma.

- Evaluar el impacto económico en las farmacias por uso de la red LF.
- Cuantificar la distancia que recorren los pacientes para obtener el medicamento una vez que ha sido reservado en otra farmacia de la red.

\section{Material y métodos}

Se trata de un estudio observacional, retrospectivo, utilizando los datos generados a través del uso de la plataforma LF durante el año 2019 (1 de enero-31 de diciembre) por 389 farmacias comunitarias en España.

LF es una plataforma IIM (Internal Inquirer Manager o Gerente de Consulta Interna) de gestión y colaboración de farmacias destinada a la mejora de la eficiencia en el control de stock. IIM proporciona un entorno colaborativo a las farmacias compartiendo información puntual sobre medicamentos concretos en el stock propio entre ellas.

La plataforma IIM tiene como objetivos principales la gestión del desabastecimiento de medicamentos. Mediante la creación de un entorno colaborativo entre las farmacias adheridas al servicio, se crea una red horizontal que pone a disposición de los pacientes información sobre la disponibilidad por proximidad geográfica de los medicamentos que necesitan.

Las premisas principales que se tuvieron en cuenta para el diseño de la plataforma IIM fueron:

- Sistema no intrusivo. La plataforma diseñada no interfiere con el resto del software utilizado por las farmacias para su gestión interna.

- Sistema autónomo. El sistema no hace uso de las funcionalidades de los diferentes sistemas informáticos de gestión de las farmacias.

- Sistema seguro. Todas las conexiones entre las diferentes farmacias de la red LF y el servidor central se realizan mediante protocolos seguros. Además, las peticiones de existencias entre farmacias se envían con los datos encriptados, asegurando la integridad de los datos entre cliente y servidor.

- Sistema con herramientas de privacidad. La plataforma cumple con la Reglamento General de Protección de Datos (RGDP) (19) y Ley Orgánica de Protección de Datos Personales y Garantía de los Derechos Digitales (LOPDPGDD) (20), evitando el almacenamiento de cualquier información personal. 
- Trabajo en tiempo real. El sistema trabaja con datos actualizados sobre la existencia de los medicamentos demandados, para asegurar la validez de la información sobre existencias.

- Información limitada. El sistema no almacena datos de stock ni de precio de los medicamentos en stock de las farmacias. Cuando se precisa de información sobre el stock de un medicamento concreto en una zona determinada, el software lanza una pregunta sobre el stock a las farmacias que la rodean, recibiendo únicamente respuesta sobre si está en stock o no (sí lo tiene/no lo tiene). De ese modo, muestra en la pantalla la farmacia más próxima que lo tiene disponible, pudiendo aumentarse o cambiar el radio de búsqueda por conveniencia geográfica. Todas las interacciones con los precios de los fármacos se realizan en tiempo real, careciendo de ninguna base de datos de precios.

De manera más concreta, lo que debe hacer el farmacéutico es seguir el proceso detallado en las diferentes pantallas de búsqueda que se detallan en las figuras 1 a 5 .

Finalmente, se genera un tique para el paciente (figura 6) que entrega en la farmacia donde finalmente se dispensa el medicamento.

Es decir:

- El farmacéutico "emisor", que realiza la búsqueda: inicia su sesión (que puede dejar abierta durante su jornada de trabajo), busca el producto, selecciona la farmacia (que es la más cercana), tramita el encargo e imprime un tique con los datos de la farmacia que ha recibido el encargo, entregando el tique $\mathrm{y}$, preferiblemente, recordando al paciente el farmacéutico, que debe acudir en 24 horas (ampliable a $72 \mathrm{~h}$ ) a recoger el encargo.

- El farmacéutico "receptor": recibe un aviso con el encargo en su pantalla del ordenador y lo acepta, tramitándolo como si hubiera sido un encargo por teléfono. En el mismo momento que se encarga le llega un aviso por lo que, simplemente, lo debe apartar y gestionarlo igual que si se lo hubieran encargado en persona o por teléfono a la espera de que cumpla con todos los requisitos en el momento de ponerlo a disposición del paciente; por tanto,

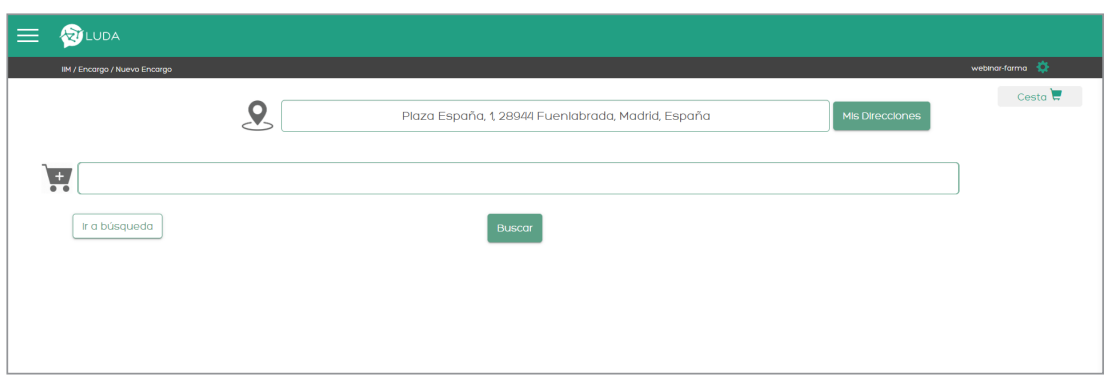

Figura 1 Proceso de búsqueda en la red 1. Barra con el buscador para introducir el producto que se busca en el stock de las farmacias que integran la red

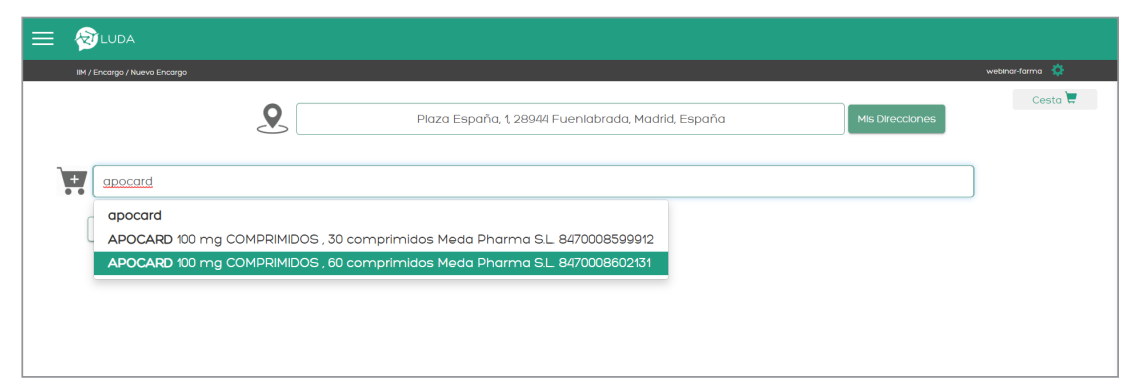

Figura 2 Proceso de búsqueda en la red 2. El buscador va ofreciendo posibilidades para facilitar la búsqueda y encontrar el producto concreto que se busca

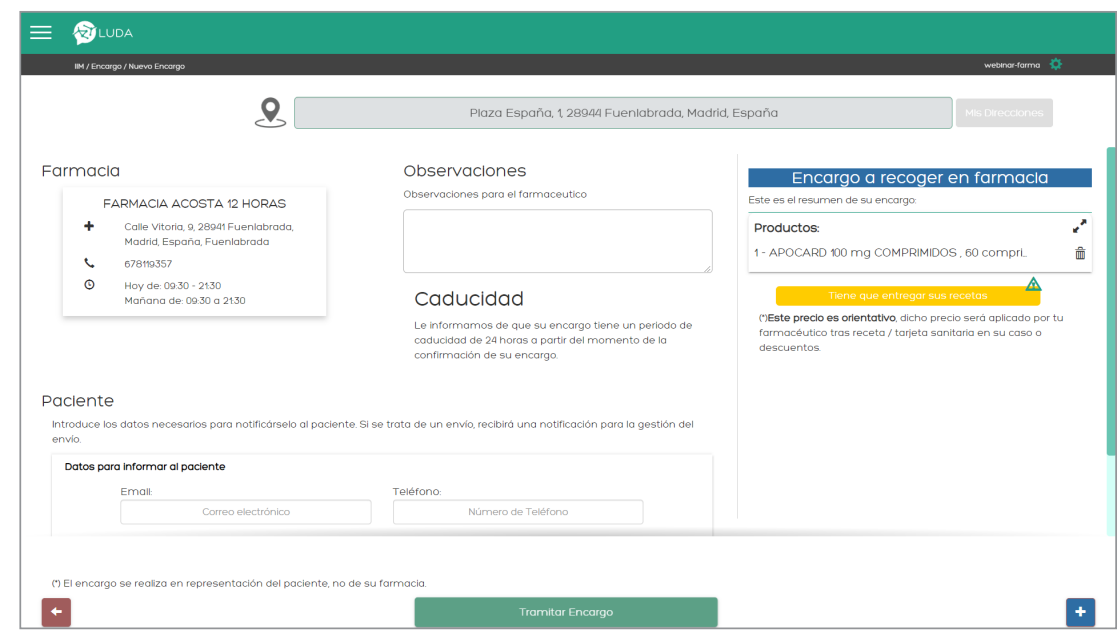

Figura 3 Proceso de búsqueda en la red 3. Una vez se ha encontrado el producto que se busca automáticamente aparece la farmacia más cercana al punto de consulta que dispone de ese producto

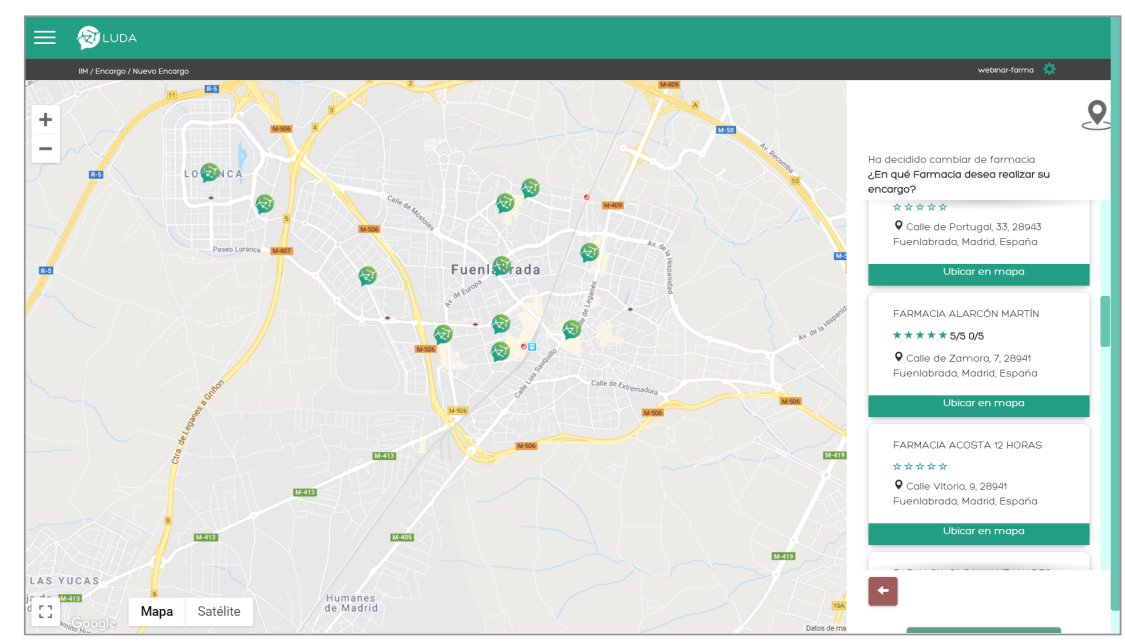

Figura 4 Proceso de búsqueda en la red 4. Si el paciente desea elegir otra farmacia puede pinchar "Elegir otra farmacia" y le aparece un mapa en el que, por orden de cercanía, aparecen las farmacias más cercanas que lo tienen. También hay un botón mediante el cual se podrian ver todas las farmacias, tengan o no el producto, sean o no de la red LF 


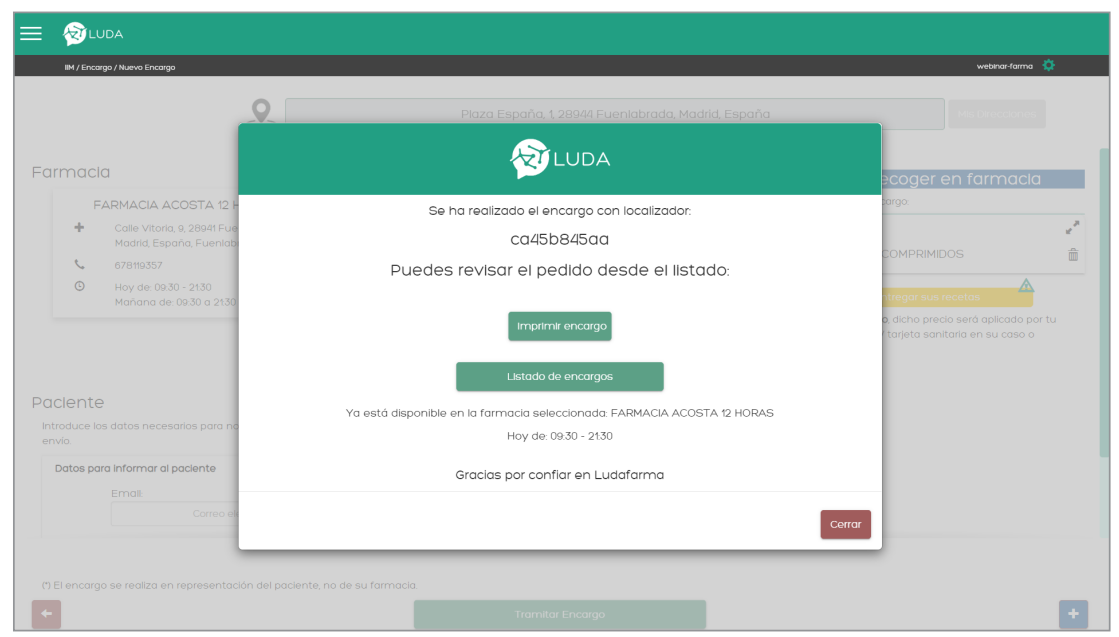

Figura 5 Proceso de búsqueda en la red 5. Una vez seleccionada la farmacia se "tramita el encargo" y la farmacia receptora recibe el aviso

no puede darse el caso de que se dispense mientras el paciente está de camino, pues ya se debería encontrar en la sección de "encargos" (o como lo gestione cada farmacia internamente). No obstante, también lo puede rechazar en ese momento, si por cualquier circunstancia ese producto estuviera ya reservado o hubiera cualquier problema de stock. Puede también cancelarlo con posterioridad o negarse a dispensarlo si, por ejemplo, detecta anomalías en la receta (o incidencias en la dispensación del medicamento que hicieran que no se pudiera dispensar) sin que esto suponga penalización de ningún tipo. Con la aceptación del encargo se da por concluido el proceso, aunque si lo desea el farmacéutico puede confirmar en el momento de la dispensación el momento exacto de la dispensación y datos de la venta. Pero para simplificar el proceso, éste concluye cuando el farmacéutico acepta el encargo.

No existen requerimientos específicos para la instalación de LF en el ordenador. Se realiza en remoto y no es necesario ningún módulo en el programa de gestión de la farmacia. LF se integra con todos los programas de gestión excepto Alquimos y, actualmente está en proceso de integración con Nixfarma. LF está plenamente integrado con Farmatic, Unycop y Farmanager y no necesita que estos programas se integren con la red por lo que la instalación es más rápida.

Por resumir en cuanto a requisitos: no necesita módulos, ni colaboración por parte de los programas de gestión, aunque todo es más fácil si es así. Solo necesita una farmacia "emisora" que busque un artículo, lo seleccione en las farmacias de la red por cercanía y una farmacia "receptora" que lo acepte. Automáticamente se genera un tique en el puesto de venta con los datos de la farmacia (contacto, horario...) a la que el paciente debe dirigirse con su receta en caso de que sea necesaria.

El coste neto para las farmacias para poder dar este servicio no obedece a cuotas. Se basa en un modelo por uso a éxito. Es decir, la farmacia emisora recibe un abono de los integrantes de la red y la que recibe, una vez ha puesto a disposición el producto que no habría dispensado si no estuviera en la red aporta una cantidad que nunca es superior a $1 €$. Es decir, cuando emite se le abona, cuando recibe factura por la dispensación del producto que recibe. Pero hay que considerar que la misma farmacia puede ser unas veces emisora y otras veces receptora, por lo que el cargo y el abono se compensan, no así lo que facture, por cuyo concepto no se paga ningún suplemento adicional.

Los medicamentos que se consideran en desabastecimiento se extraen de los listados CISMED y de la AEMPS. Se comparan ambos listados por dos razones: validación y homogenización. En España ambos listados arrojan resultados diferentes pues se obtienen mediante metodologías (descritas antes) diferentes, por lo que si queremos mostrar resultados de efectividad de LF en el impacto de los desabastecimientos entendemos que se deben incluir los dos métodos. Ambos listados son los de referencia

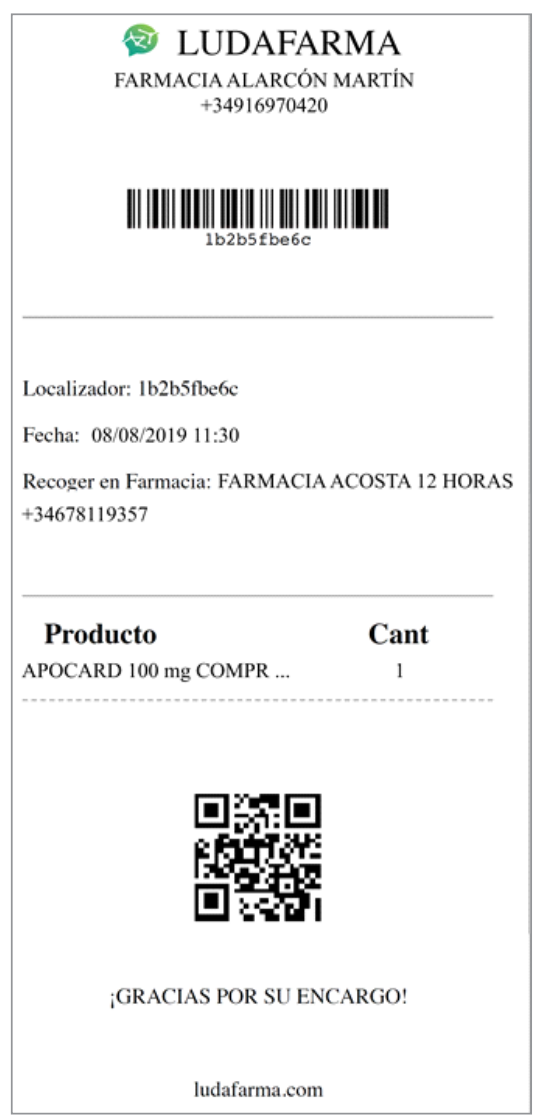

Figura 6 Tique generado a paciente para recogida del medicamento

que se usan en España en el análisis de desabastecimientos, y se usan para que sea más fácil explicar la universalidad de resultados y conclusiones.

Las peticiones de medicamentos realizadas semanalmente a través de la red LF se cruzaron con la base de datos de actualización semanal que aporta el sistema CISMED (16), señalando los medicamentos que se solicitaron a través de dicha red y que se encontraban en los listados de medicamentos con problemas de suministro CISMED.

Las peticiones realizadas a través de la red LF se cruzaron el 31 de diciembre de 2019 con el listado de medicamentos con problemas de suministro que proporciona la AEMPS. En este listado, si la consulta es el 31 de diciembre, solo están los medicamentos con problemas de suministro en ese momento y los medicamentos cuyo problema de suministro se ha resuelto en el segundo semestre. Por otro lado, no están aquellos medicamentos cuyo problema de suministro se resuelve antes del 1 de julio de 2019 (en el primer semestre del 2019) (21). La única manera de tener información sobre medicamentos que 
estuvieron con problemas de suministro por semestres anteriores a la fecha de la consulta es acceder a unos informes que son en realidad resúmenes (22). Es decir, el 31 de diciembre de 2019 no fue posible consultar datos del primer semestre de los medicamentos cuya falta de suministro se ha resuelto antes del 1 de julio porque no están accesibles en la web de la AEMPS.

La nomenclatura empleada para realizar el cruce de los medicamentos fue el CN (código nacional), un código único asignado y registrado en la AEMPS para cada presentación. (Anexo I: medicamentos buscados en LF en las fechas del estudio).

Los datos agregados (codificados y anónimos) se extrajeron con codificaciones que cambian cuando se vuelve a solicitar la misma información a LF. Dicha información no se almacena en ningún servidor.

Toda la información se procesó con el lenguaje de programación R (23) (versión 3.6.2), qué está orientado al análisis de datos y estadística computacional, junto con el IDE (entorno de desarrollo integrado) para RStudio (versión 1.2.5033). Para la gestión de fechas se utilizó el paquete Lubridate. Se usó esta tecnología porque permite aportar reproducibilidad en el análisis estadístico, generando scripts (archivos de secuencia de comandos), que pueden ejecutar todas las operaciones llevadas a cabo a lo largo del estudio, tanto con fines retrospectivos (revisión del análisis y su interpretación), como prospectivos (futuros estudios). De esta manera se obtiene la posibilidad de unir y cruzar varios archivos de fuentes distintas -información encriptada de la red LF y archivos públicos Excel de CISMED (16) y AEMPS (21)-. El script permite tabular de manera que concuerde cada fila o columna de los distintos archivos.

Todos los listados de medicamentos con falta de suministro publicados en CISMED durante el año 2019 se descargaron, unificaron y convirtieron a texto plano (csv, valores separados por coma). En términos generales el script que cruzó ambas tablas cargó en memoria la base de datos de CISMED previamente unificada y la de LF, generó un listado de medicamentos en desabastecimiento y cuantificó cuántos encargos de LF se correspondían con cada medicamento con problemas de suministro dentro de las fechas en la que estaba comprendida esa situación desfavorable informada por CISMED.

Finalmente se descargó la documentación sobre problemas de suministros activos a fecha del estudio y resueltos en los últimos seis meses, publicada por la AEMPS en CIMA (Centro de Información de Medicamentos AEMPS). Se convirtió esa tabla a texto plano (csv, valores separados por coma) para facilitar el análisis estadístico.

Para comparar ambos listados se generaron intervalos de fechas, tanto para cada encargo individual de LF (una única fecha genera un intervalo) como para los intervalos de fechas de desabastecimientos publicados por CISMED, pues este listado ofrece resultados semanales, mientras que para la AEMPS se consideró fecha de inicio del encargo y fecha prevista de fin del desabastecimiento, pues la AEMPS asume periodos de desabastecimiento. En aquellos casos en los que la AEMPS no contemplaba aun una fecha de finalización del problema de suministro (porque el medicamento en la fecha del encargo estaba en desabastecimiento y en la fecha en que se realizó este estudio seguía estando así en ese listado) se consideró la fecha de lanzamiento del script como fecha prevista de fin del problema. A partir de este punto se cuantificaron cuántos encargos de LF se correspondían con cada medicamento con problemas de suministro dentro de las fechas en la que estaba comprendida esa situación desfavorable informada por la AEMPS. Es decir, para poder comparar cronológicamente los datos públicos y los de LF convertimos todos los datos de fechas a intervalos de fechas.

- En el caso de LF se dispone de la fecha de creación del encargo, con el formato AAAA-MM-DD HH:MM: SS (año-mes-día hora: minutos: segundos). Se convierte por tanto a AAAAMM-DD-AAAA-MM-DD resultando un intervalo de tiempo de 24 horas correspondiente al día del encargo para poder integrar bases de datos. Es decir, lo que hacemos es convertir un momento concreto en el tiempo en un intervalo de tiempo correspondiente a un día dado para poder cruzarlo fácilmente con los periodos de tiempo que utilizan las otras bases de datos, puesto que para poder manejar grandes cantidades de datos (big data) es necesario automatizar la respuesta que requiere el tratamiento; al cruzar diferentes de bases de datos estas deben tener el mismo formato.

- En el caso de la AEMPS se dispone de dos fechas: la fecha de inicio del problema de suministro y la fecha de fin del problema de suministro. En ocasiones no hay fecha de fin de suministro $\mathrm{y}$, por tanto, consideramos que en el momento de lanzar el script aún persiste el problema considerando ese día la fecha de fin del problema y procedemos a la conversión como el punto anterior.

- En el caso de CISMED trabajamos con las fechas en las que se recogen sus resultados que son equivalentes a una semana y procedemos igual que los puntos anteriores.

A partir de este punto se cuantificaron cuántos encargos de LF se correspondían con cada medicamento con problemas de suministro dentro de las fechas en las que estaba comprendida esa situación desfavorable.

Se consideraron para el estudio únicamente "encargos concluidos con éxito", aquellos en los que se formalizó el encargo a través de la red LF y el paciente acudió a retirar su medicación (independientemente de que estuvieran reconocidos como con problemas de suministro por la AEMPS o por CISMED).

En resultados, para facilitar su exposición, se listan los 22 medicamentos más buscados, ya que 22 es el punto de corte en el que vimos que cambia de manera más acusada la frecuencia de resultados en los datos (se empieza a perder significación en la frecuencia). Este mismo razonamiento se sigue para exponer más fácilmente los resultados del anexo IV, en el que el punto de corte se establece en la línea 20.

Las distancias recorridas y los tiempos empleados por los pacientes para recoger las reservas de medicamentos realizadas a través de LF se midieron a través de Distance Matrix API (Application Program Interface) de la aplicación Google Cloud Platform $^{\odot}$. Esta API de matriz para distancias es un servicio que proporciona distancia y tiempo de viaje para una matriz de orígenes y destinos. La API devuelve información basada en la ruta recomendada entre los puntos de inicio y finalización, calculada por la API de Google Maps, y consta de filas que contienen valores de duración y distancia para cada par. 


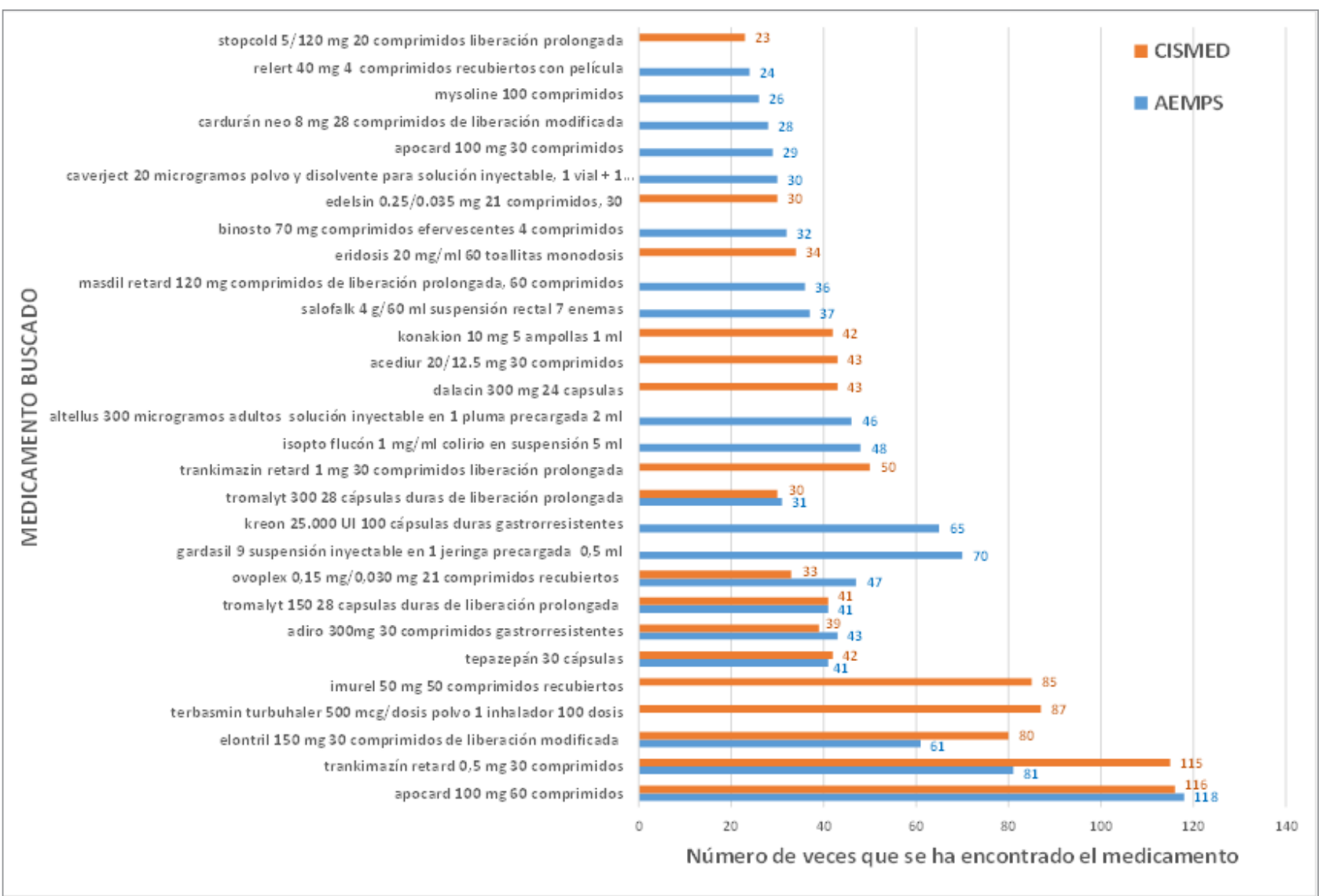

Figura 7 Medicamentos en desabastecimiento encontrados a través de la red LF

\section{Resultados}

Participaron 389 farmacias comunitarias adheridas libre y gratuitamente a la red LF que realizaron 7.628 encargos concluidos con éxito. De este total de encargos, 1.993 correspondieron a medicamentos con problemas de suministro según los listados de la AEMPS (anexo II) y 1.794 a medicamentos con problemas de suministro según los listados de CISMED (anexo III).

De los medicamentos que se encontraron a través de la red LF había 245 presentaciones que estaban en el listado AEMPS y 168 presentaciones que estaban en el listado CISMED.

De los medicamentos reconocidos con problemas de suministro por la AEMPS o por CISMED y que se reservaron a través de la red LF, el CN 860213 (Apocard $^{\circledR}$ 100mg 60 comp) fue el más solicitado teniendo en cuenta los dos listados (118
AEMPS/116 CISMED), seguido del CN

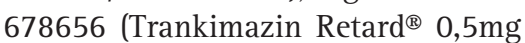
30 comp) (81/115) y del CN 658760 (Elontril ${ }^{\circledR} 150 \mathrm{mg} 30$ comp) (61/80), seguidos del CN 692382 (Terbasmin turbuhaler $500 \mathrm{mcg} /$ dosis polvo 1 inhalador 100 dosis) (87) (CISMED) y del CN 933176 (Imurel 50 mg 50 comprimidos recubiertos) (85) (CISMED) y del CN 658978 (Gardasil 9 suspensión inyectable en 1 jeringa precargada 0,5 ml)(70) (AEMPS) y del CN 672971 (Kreon 25.000 UI 100 cápsulas duras gastrorresistentes) (65) (AEMPS).

Los medicamentos que se encargaron a través de la red LF más de 22 veces y que se encontraban en los listados de medicamentos con problemas de suministro de la AEMPS y de CISMED se recogen en la figura 7.

En el anexo IV se detallan todos los encargos realizados con éxito de la red LF (7.628), ordenados por encargos solicitados (las operaciones que generan las farmacias que más búsquedas solicitan) y por encargos recibidos (las farmacias que más encargos reciben). La facturación de las 389 farmacias en el año de estudio por el uso de la red LF arrojó valores con una alta dispersión. La facturación aumentó un promedio de $414,24 €(D E=596,81)$. El valor máximo de facturación de las farmacias analizadas gracias al uso de la red LF fue de 4.537,70 €, el 75\% 545,76€, $50 \% 173,99,25 \% 144,91 €$ y el valor mínimo 0,1694 €. En ningún caso hubo valores negativos.

Las distancias recorridas y el tiempo empleado estimado por los ciudadanos que encontraron los medicamentos que necesitaban a través de la red LF se reflejan en los anexos $V_{-} 1$ y V_2 según listado AEMPS y CISMED respectivamente, y se reflejan agrupadas por distancias en la tabla 1 (distancias y tiempos empleados en recorrerlos a

Tabla 1 Distancias y tiempos empleados en recorrerlos a pie entre farmacias que emiten y reciben operaciones de medicamentos de los listados AEMPS (Encargos: 1.993)

\begin{tabular}{|l|c|c|c|c|c|c|}
\hline & Valor mínimo & $\mathbf{2 5} \%$ & $\mathbf{5 0} \%$ & $\mathbf{7 5} \%$ & Valor máximo & Media \\
\hline Distancia (metros) & 63 & 912 & 1.870 & 4.654 & 523.504 & 7.813 \\
\hline Tiempo (segundos) & 51 & 688 & 1.429 & 3.525 & 391.080 & 5.847 \\
\hline
\end{tabular}


Tabla 2 Distancias y tiempos empleados en recorrerlos a pie entre farmacias que emiten y reciben operaciones de medicamentos de los listados CISMED (Encargos: 1.794)

\begin{tabular}{|l|c|c|c|c|c|c|}
\hline & Valor mínimo & $\mathbf{2 5} \%$ & $\mathbf{5 0} \%$ & $\mathbf{7 5} \%$ & Valor máximo & Media \\
\hline Distancia (metros) & 53 & 676 & 1.493 & 3.014 & 523.504 & 5.129 \\
\hline Tiempo (segundos) & 36 & 509 & 1.130 & 2.251 & 391.080 & 3.847 \\
\hline
\end{tabular}

pie entre farmacias que emiten y reciben operaciones de medicamentos de los listados AEMPS), en la que se puede ver que la distancia media es de $7,8 \mathrm{~km}, \mathrm{DE}=370,13$ y en la tabla 2 (distancias y tiempos empleados en recorrerlos a pie entre farmacias que emiten y reciben operaciones de medicamentos de los listados CISMED) en la que se puede ver que la distancia media es de 5,1 km, DE $=370,14$.

\section{Discusión}

El problema de falta de suministro de ciertos medicamentos es una de las incidencias a las que el farmacéutico, tanto hospitalario como comunitario, debe enfrentarse hoy en día. Frente a ello la red LF, pionera en España, ofrece la oportunidad de amortiguar el efecto de la interrupción de la puesta a disposición de ciertas presentaciones de medicamentos en las farmacias para que estas lo dispensen a los ciudadanos. LF permite conocer en tiempo real si un medicamento se encuentra disponible en una farmacia del entorno al punto de consulta, ampliando el radio de puntos de stock a los que consulta por geolocalización y sin otro criterio más que la distancia más próxima a la farmacia que origina la consulta.

Aunque el número de farmacias participantes en el estudio ha sido pequeño, 2 \% sobre el total del número de farmacias de la red nacional, se han resuelto una media de diez casos en cada una de ellas, lo que nos hace pensar que, a mayor número de farmacias adheridas, mayor podría ser el número de solicitudes y, por tanto, mayor probabilidad de solucionar casos de medicamentos en desabastecimiento.

Respecto a los medicamentos con problemas de suministro durante el año de estudio, el CN 860213 (Apocard $^{\circledR} 100 \mathrm{mg} 60$ comp) ha sido el más solicitado en cualquiera de los dos listados (AEMPS y CISMED), medicamento que tenía una difícil sustitución terapéutica, seguido del $\mathrm{CN}$
678656 (Trankimazin Retard $^{\circledR} 0,5 \mathrm{mg}$ 30 comp), que no disponía de alternativa con la misma forma farmacéutica, y del CN 658760 (Elontril $^{\circledR} 150 \mathrm{mg}$ 30 comp), que a pesar de disponer de medicamentos para su sustitución, estos no se encontraban tampoco disponibles durante el periodo de estudio.

Hay que resaltar también los casos en los que se solicitó el CN 841056 (Adiro ${ }^{\circledR} 300 \mathrm{mg} 30$ comp), que no disponía de ninguna presentación para su sustitución o del CN 692382 (Terbasmin $\mathrm{TH}^{\circledR} 500 \mathrm{mcg} /$ dosis 100 dosis), que es un medicamento insustituible según la legislación vigente. Otros CN sin posibles alternativas para realizar sustituciones por el farmacéutico fueron: 656714 (Altellus $^{\circledR}$ $300 \mathrm{mcg} / 0,3 \mathrm{~mL}$ ) o 707342 (Gardasil $9{ }^{\circledR}$ jeringa precargada).

Otro aspecto importante para las farmacias es su propia viabilidad. Una de las opciones que se manejan para potenciarla es el pago por servicios nuevos (24) o por algunos de los servicios que tradicionalmente ha venido prestando la farmacia comunitaria, como las guardias nocturnas, que podían soportar contra su margen en épocas en las que las farmacias no tenían una política fiscal tan dura que implica descuentos por volumen de facturación $(25,26,27)$ a las administraciones autonómicas y nacionales o una gestión de la "factura farmacéutica”, basada en rebajas de margen (28) y PVP. Estos nuevos pagos por servicios no se materializan, pero integrarse en la red LF supone poder dar un servicio tecnológico muy avanzado que aporta soluciones reales y rápidas para un problema muy serio para el paciente y para la perspectiva de la eficiencia del sistema sanitario español, con el grave impacto sobre los gastos económicos, el número de errores de medicación, las reacciones adversas, la mortalidad y las quejas de los pacientes. El sistema colaborativo de LF hace que el uso de esta herramienta en ningún caso suponga desembolso o inversión por parte de la farmacia o del ciudadano; es más, las farmacias que más solicitudes a éxito realizan tienen un abono por uso de la red que es en realidad aportado por todos los miembros de ella. Por otro lado, la farmacia que recibe el encargo va a poder dar un servicio que concluye con la dispensación y cobro del medicamento que no habría dispensado, ya que el paciente le ha encontrado gracias a la red.

Respecto a la facturación todas las farmacias aumentaron en mayor o menor medida la facturación, si bien la dispersión de los datos es muy alta; es decir, la desviación estándar es mayor que la media, dado que tenemos valores muy extremos pues la facturación depende del número de operaciones que cada farmacia realiza. En ningún caso el uso de la red supuso facturación negativa. Analizándolo a través de los cuartiles, el $75 \%$ de las farmacias ha facturado 545,76 € o menos (hasta $0,17 €$ ) o viéndolo de otra forma, hubo un $25 \%$ de farmacias que facturaron $545,76 €$ o más (hasta 4.537,70 € ).

A pesar de que la mayoría de los ciudadanos encontraron el medicamento a unos tres kilómetros de la farmacia en la que hicieron la petición, cuantas más farmacias haya adheridas a la red, mayor stock se compartiría y el número de operaciones a menor distancia del punto de consulta podría ser mayor, pues LF prioriza para el posicionamiento de resultados solo la cercanía de producto buscado al paciente. En concreto para AEMPS el $75 \%$ de los encargos está por debajo de 4,65 kilómetros. Para CISMED el $75 \%$ de los encargos está por debajo de 3,01 kilómetros.

Así, los promedios de distancia a los que se encuentran los medicamentos son de 7,8 km para medicamentos en los listados con problemas de suministro de la AEMPS y 5,1 km para medicamentos en los listados con problemas de suministro de CISMED.

Si el $14,7 \%$ de la población española refiere sufrir una limitación física para andar (13), limitando esta dificultad su capacidad de movilidad a 
estos pacientes a 300 metros, lo que es poco más de los $250 \mathrm{~m}$ a los que con carácter general puede encontrar otra farmacia, la red LF les permite acceder fácilmente a la medicación en falta de suministro y que necesitarian a 300 $m$ en 69 de las reservas a través de la red LF de medicamentos con problemas de suministro, según la AEMPS (3,4\% de dichas reservas) y 90 de las reservas a través de la red LF de medicamentos con problemas de suministro según CISMED (5\% de dichas reservas). Si se aumentara el número de farmacias, la población frágil tendría más posibilidades de encontrarlo dentro de su capacidad de movilidad.

A la vista de los resultados creemos que pudiera ser de gran utilidad la implantación de una red LF con más farmacias, y añadir un sistema de entrega directa de medicamentos a los pacientes por las farmacias, especialmente para aquellos que presentan problemas de movilidad. También se podría plantear la posibilidad de que, aunque se facture por la farmacia receptora del encargo, la entrega se realizara por parte de la emisora del encargo; por ejemplo, en el caso de que el fuera un paciente vulnerable.

Cabe especificar cómo debemos interpretar en este estudio la dispersión de algunas secuencias de datos, concretamente la de las distancias. Consideramos que es útil expresar cuartiles: la dispersión es alta, ya que LF se usa para encontrar medicamentos con una gran variación de distancias (los pacientes llegan a hacer recorridos muy largos para acceder al medicamento que necesitan, incluso a otras comunidades autónomas). Así, que el medicamento que se necesita esté muy lejos, no limita la utilidad de LF como habríamos podido pensar a priori.

Por último, sería interesante para futuros estudios analizar los datos de acceso a medicamentos con problema de suministro de todo 1 año según la AEMPS, ya que en el presente estudio solo fueron accesibles los datos del último semestre de 2019 .

\section{Conclusiones}

La red LF ha permitido encontrar durante el año 2019 hasta casi 4.000 veces medicamentos que se encontraban en falta de suministro.

Apocard $^{\Phi} 100 \mathrm{mg} 60$ comprimidos, que no puede ser sustituido por otro medicamento según la legislación vigente, y que su indicación es la taquicardia auricular, ha sido el medicamento más veces localizado a través de la red, seguido de Trankimazin Re$\operatorname{tard}^{\oplus} 0,5 \mathrm{mg}$ que no tiene alternativa con la misma forma farmacéutica.

La red LF ayuda a los pacientes y a la eficiencia del sistema sanitario por disminuir el impacto del desabastecimiento en el incumplimiento terapéutico.

La red LF ayuda a los farmacéuticos en la medida que les permite dar una solución in situ a los pacientes que no encuentran los medicamentos que buscan y aumenta la facturación de las farmacias sin realizar ningún desembolso. Indirectamente ayuda al sistema sanitario disminuyendo los costes debidos a la pérdida de horas en resolver problemas de suministro y sobrecostes por otras alternativas a los medicamentos en desabastecimiento.

En la mayoría de las veces estos medicamentos se han encontrado a una distancia inferior a los $3 \mathrm{~km}$, empleándose aproximadamente una media de entre una hora y hora y media para encontrar los medicamentos en desabastecimiento.

En 10 casos según listados AEMPS y en 7 casos según CISMED la distancia a recorrer por los pacientes para adquirir su medicamento con problemas de suministro fue superior a $300 \mathrm{~km}$.

\section{Agradecimientos}

Queremos agradecer a todas las farmacias que están adheridas a la red LF, y a los farmacéuticos comunitarios que en ellas trabajan, el uso de la red que ha permitido elaborar este estudio.

\section{Referencias bibliográficas}

1. Problemas de suministro de medicamentos [Sede web]. Madrid: AEMPS: 2019. [Acceso 11 de febrero de 2020]. Disponible en: https://www.aemps. gob.es/medicamentosUsoHumano/ problemasSuministro/home.htm

2. EU regulatory network reflection paper on the availability of authorised medicinal products for human and veterinary use. Amsterdam: EMA; 2018. [Acceso 11 de febrero de 2020]. Disponible en: https://www.hma.eu/ fileadmin/dateien/HMA_joint/00-_ About_HMA/03-Working_Groups/ TF_Availability/2018_08_TF_AAM_ Reflection_paper_on_the_availability_ of_authorised_medicinal_products_ for_human_and_veterinary_use.pdf

3. Drug shortages roundtable: Minimizing the impact on patient care. Am J Health-Syst Pharm. 2018; 75:816-20. https://doi.org/10.2146/ajhp180048

4. Fox ER, Tyler LS. Managing drug shortages: seven years' experience at one health system. Am J Health Syst Pharm. 2003; 60(3):245-253. https:// doi.org/10.1093/ajhp/60.3.245

5. McLaughlin M, Kotis D, Thomson K, Harrison M, Fennessy G, Postelnick M, Scheetz MH. Effects on patient care caused by drug shortages: a survey. J Manag Care Pharm. 2013; 19(9):783-8. https://doi.org/10.18553/ jmcp.2013.19.9.783

6. Pauwels K, Huys I, Casteels M, Simoens S, Drug shortages in European countries: a trade-off between market attractiveness and cost containment?. BMC Health Serv Res. 2014; 14: 438. [cited 2020 Jun 5];14(1). Disponible en: https://bmchealthservres.biomedcentral.com/articles/10.1186/1472-6963-14-438

7. Ventola MS. The Drug Shortage Crisis in the United States. Causes, Impact, and Management Strategies. Pharmacy and Therapeutics. 2011; 36(11): 740-757. https://www.ncbi.nlm.nih. gov/pmc/articles/PMC3278171/

8. Woodcock J. Drug Shortages: Root Causes and Potential Solutions A Report by the Drug Shortages Task Force. FDA News Release. 2019. [acceso 1 de febrero de 2020]. Disponible en: https://www.fda.gov/media/131130/ download

9. Phuong JM, Penm J, Chaar B, Oldfield LD, Moles R. The impacts of medication shortages on patient outcomes: A scoping review. PLoS ONE.2019; 14(5). https://doi.org/10.1371/journal. pone. 0215837

10. Said A, Goebel R, Ganso M, Zagermann-Muncke P, Schulz M. Drug shortages may compromise patient safety: Results of a survey of the reference pharmacies of the Drug Commission of German Pharmacists. Health Policy. 2018; 122: 1302-1309. https://doi.org/10.1016/j.healthpol.2018.09.005

11. Kaakeh R, Sweet BV, Reilly C, Bush C, De Loach S. Impact of drug shortages on U.S. Health systems. Am J Health Syst Pharm. 2011; 68: 1811-1819. https://doi.org/10.2146/ajhp110210

12. AEMPS. Plan de Garantías de Abastecimiento de Medicamentos 20192022. Ministerio de Sanidad, Consumo y Bienestar Social; 2019 [acceso 1 de febrero de 2020]. Disponible en: https://www.aemps.gob.es/medicamentosUsoHumano/problemasSuministro/docs/plan-garantias-abastecimiento-AEMPS-2019-2022.pdf

13. Physical and sensory functional limitations by sex, age and educational attainment level [Sede web]. Luxemburgo: Eurostat; 2020. [Acceso 
13 de febrero de 2020]. Disponible en: https://appsso.eurostat.ec.europa. eu/nui/show.do?dataset=hlth_ehis_ pl1ectlang $=e n$

14. Prevalence of Disability and Disability Type Among Adults - United States. [Sede web]. Washington: CDC; 2015. [Acceso 13 de febrero de 2020]. Disponible en: https://www. cdc.gov/mmwr/preview/mmwrhtml/ mm6429a2.htm

15. Ley 16/1997, de 25 de abril, de regulación de servicios de las oficinas de farmacia. Boletín Oficial del Estado, $\mathrm{n}^{\circ} 100$ [Internet]. 1997 [Acceso $13 \mathrm{de}$ febrero 2020]. Disponible en: https:// www.boe.es/buscar/doc.php?id=B0E-A-1997-9022

16. Centro de Información sobre el Suministro de Medicamentos (CISMED). Listado de medicamentos con problemas de suministro [sede web]. Madrid: CGCOF; 2019 [acceso 4 de enero de 2020]. Disponible en: https://www. portalfarma.com/Profesionales/medicamentos/CISMED/Paginas/Listados-Medicamentos-problemas-suministro.aspx

17. Pharmaceutical Group of European Union. PGEU Medicine Shortages Survey 2019 Results. [sede web]. Brussels: PGEU; 2019. [Acceso 4 de enero de 2020]. Disponible en: https:// www.pgeu.eu/publications/press-release-pgeu-medicine-shortages-survey-2019-results/

18. Martín R, Acosta JA. Tecnología, desabastecimiento y rentabilidad de un nuevo servicio para las farmacias (Comunicación Póster). 31 Congreso Europeo de Oficina de Farmacia y Sa- lón de Medicamentos y Parafarmacia. Barcelona: COFB-COFM; 2019.

19. Reglamento (UE) 2016/679 del Parlamento Europeo y del Consejo, de 27 de abril de 2016, relativo a la protección de las personas físicas en lo que respecta al tratamiento de datos personales y a la libre circulación de estos datos y por el que se deroga la Directiva 95/46/CE (Reglamento general de protección de datos). Diario Oficial de la Unión Europea, no L 119 (04-052016). [Acceso 2 de febrero de 2020]. Disponible en: https://www.boe.es/ doue/2016/119/L00001-00088.pdf

20. Ley Orgánica 3/2018, de 5 de diciembre de Protección de Datos Personales y garantía de los derechos digitales. BOE, no 294 (06-12-2018). [Acceso 2 de febrero de 2020]. Disponible en: https://www.boe.es/buscar/doc.php?i$d=$ BOE-A-2018-16673

21. AEMPS. Listado de medicamentos que tienen actualmente problemas de suministro. [Acceso 31de diciembre 2019]. Disponible en: https://cima. aemps.es/cima/publico/listadesabastecimiento.html?activos $=1$

22. AEMPS. Informe semestral sobre problemas de suministro (julio-diciembre 2019). Madrid: AEMPS; 2020. [Acceso 2 de febrero de 2020]. Disponible en: https://www.aemps.gob. es/distribucion-de-medicamentos/ problemas-de-suministro-de-medicamentos/

23. $\mathrm{R}$ Core Team (2019). R: A language and environment for statistical computing. R Foundation for Statistical Computing, Vienna, Austria. Disponible en: https://www.R-project.org/
24. Baixauli VJ, Satué-de-Velasco E, Gil MI, Roig JC, Villasuso B, Sáenz-de-Buruaga S. Propuesta de la Sociedad Española de Farmacia Comunitaria (SEFAC) sobre servicios profesionales farmacéuticos en farmacia comunitaria. Farmacéuticos Comunitarios. 2013 Sep 30; 5(3):119-126.

25. Antares Health Lines [Sede web]. Madrid: Antares Consulting; 2015. [Acceso 2 de febrero de 2020]. Situación y cifras claves de la farmacia en 2014. [pp 10, 15, 16, 17-26]. Disponible en: https:// www.antares-consulting.com/uploads/ TPublicaciones/91b3fb35d40d4bbc984800252bec5f8937908a25.pdf

26. Grupo Mensor Servicios de salud. La realidad económica de la farmacia en España: Estudio del impacto económico en las oficinas de farmacia de las medidas de contención del gasto en medicamentos en los últimos diez años. CGCOF. La distribución de la farmacia en España. Madrid: Mensor Servicios de Salud; 2011. [Acceso 7 de julio de 2020]. Disponible en: http://cofsegovia.portalfarma.com/ Documentos/Portada/MENSOR_Enero2011[1].pdf

27. Besalduch JM. Informe Anual de Oficinas de Farmacia (XX edición). Madrid: Aspime SL; 2019.

28. Cobián Rodríguez MB, Martínez Romero F, Murillo Fernández MD, Sanz Granda A, Satué de Velasco E, Baixauli Fernández VJ. Propuesta de sistema retributivo de SEFAC para la prestación del servicio de dispensación al Sistema Nacional de Salud. Farmacéuticos Comunitarios 2012; 4(4): 144-164. 